\title{
La doble evaluación: un medio para aprender de los errores y para mejorar el rendimiento en matemáticas
}

\author{
Francisco J. Boigues ${ }^{a}$, Vicente D. Estruch ${ }^{\mathrm{b}}$, Anna Vidal ${ }^{\mathrm{c}}$ y José I. Pastor ${ }^{\mathrm{d}}$ \\ Departament de Matemàtica Aplicada, Universitat Politècnica de València-Campus de Gandia \\ Grup d’Innovació Educativa i Recerca en Màteries Cientifiques \\ afraboipl@mat.upv.es, bvdestruc@mat.upv.es, cavidal@mat.upv.es,..jpastogi@mat.upv.es
}

\begin{abstract}
This paper presents an educational innovation in the field of mathematics consisting of implementing a "double assessment" system (DDE). Any assessment in teaching should not only measure the acquisition of knowledge in the broadest sense, but also should help overcome the obstacles and difficulties experienced during learning. In the double evaluation, students face a first test of control and, within a few days, they face another test with the same learning objectives. In the time interval between the two tests, in class, those contents are worked especially where greater learning deficiencies have been observed. The experience has been carried out during the first semester of the 2017/2018 academic year and 61 first-degree students from the Degree in Environmental Sciences of the Polytechnic University of Valencia participated. The results of the experience show significant improvements in the performance of the students and in addition there is a greater implication of the students in their own learning.
\end{abstract}

Keywords: Double-Assessment, feedback, mathematics, innovation.

\section{Resumen}

Este trabajo presenta una innovación educativa en el campo de las matemáticas consistente en implementar un sistema "De Doble Evaluación" (DDE). Cualquier evaluación en docencia no solo debe medir la adquisición de conocimientos en sentido amplio; sino que además debería ayudar a superar los obstáculos y dificultades experimentados durante el aprendizaje. En la doble evaluación, los estudiantes se enfrentan a un primer examen de control y, a los pocos días, se vuelven a enfrentar a otro examen con los mismos objetivos de aprendizaje. En el intervalo de tiempo entre las dos pruebas, en clase, se trabajan especialmente aquellos contenidos donde se han observado mayores deficiencias de aprendizaje. La experiencia se ha llevado a cabo a lo largo del primer cuatrimestre del curso 2017/2018 y participaron 61 estudiantes de primer curso del Grado de Ciencias Ambientales de la Universitat Politècnica de València. Los resultados de la experiencia demuestran mejoras significativas en el rendimiento de los estudiantes y además se constata una mayor implicación de los estudiante en su propio aprendizaje. 
La doble evaluación: un medio para aprender de los errores y para mejorar el rendimiento en matemáticas

Palabras clave: Doble-Evaluación, realimentación, matemáticas, innovación.

“Quien nunca ha cometido un error, nunca ha probado algo nuevo.”

A. Einstein

\section{Introducción}

El rendimiento académico de los estudiantes universitarios depende de muchos y diversos factores: los conocimientos previos, los hábitos de estudio, la motivación, las metodologías predominantes, el sistema de evaluación, etc. Consecuentemente, analizar dichos factores, en el sentido de valorar su influencia en el rendimiento final, ayudará a promover mejoras en los sistemas de enseñanza/aprendizaje universitarios, que conllevarán necesariamente progresos adecuados en el rendimiento (Myers y Myers, 2007; Mourshed, Chijioke y Barber,2010; Zabalza,2004).

De entre los factores mencionados, uno de los que condicionan de manera significativa la forma en que los estudiantes estudian determinada materia académica es el método de evaluación (Gargallo, 2015).

Existen diversas maneras de entender la evaluación, dependiendo de las necesidades, propósitos u objetivos de la institución educativa, como pueden ser, por ejemplo, el control y la medición, el enjuiciamiento de la validez del objetivo o la rendición de cuentas (Isabel y Vargas, 2004). Los modelos Tylerianos (Tyler, 1974), que siguen plenamente vigentes en la actualidad, ponen especial atención en los objetivos relacionados con el rendimiento de los estudiantes. Para estos modelos, la evaluación debe medir hasta qué punto han sido alcanzados los objetivos de aprendizaje. Para ello, deben establecerse mecanismos que permitan comparar los resultados de aprendizaje frente los objetivos, establecidos éstos de antemano y, asimismo, propuestos en los planes de estudios (Tyler, 1974, Hernández, 2010). Por lo tanto, uno de los objetivos de la evaluación será obtener una medida de los conocimientos adquiridos. En matemáticas, cuando nos referimos a los conocimientos adquiridos, éstos no se concretan únicamente en aspectos teóricos como las definiciones, las proposiciones o las propiedades. También son muy importantes los algoritmos de cálculo, así como la aplicación de los conocimientos a la resolución de problemas. Estos tres aspectos (teoría-práctica-aplicabilidad) constituyen la base de lo que se entiende actualmente como competencia matemática (BOE 1105/2014).

Myers y Myers (2007) encontraron que aquellos estudiantes que realizaron exámenes o controles a menudo obtuvieron mejores resultados académicos en matemñaticas que otros estudiantes que realizaron los mínimos controles posibles. Además, aquellos estudiantes que tuvieron más controles manifestaron una actitud más positiva frente a la clase de matemáticas. Pero existe un límite ya que evaluaciones muy frecuentes, por ejemplo exámenes semanales, no conduce a mejores resultados (Haberyan, 2003). Por lo tanto, hay que buscar un equilibrio a la hora de programar actos de evaluación, teniendo en cuenta

(cc) EY-NC-ND 2018, Universitat Politècnica de València

Congreso IN-RED (2018) 
también que los estudiantes necesitan cierto margen para manejar y gestionar su tiempo y sus estrategias de estudio.

Toda evaluación efectiva ha de tener en cuenta la dimensión formativa, es decir informar, tanto al profesor como al alumno, sobre los avances en el proceso de enseñanza/aprendizaje. Algún trabajo reciente (Fabregat y Pelayo, 2016) reivindica que la dimensión formativa de la evaluación debería tener mayor consideración en la práctica docente. En la faceta formativa, la retroalimentación ("feedback") debe ser una componente esencial (Sadler y Good,2006). El feedback ha de permitir que el estudiante sea consciente de lo que ha realizado bien y de lo que ha realizado mal, y además que pueda identificar las causas de sus errores, es decir identificar las estructuras cognitivas que son deficientes (Simon y Tzur, 2004). Como conclusión a lo expuesto, parece razonamble asumir que hay que poner en práctica modelos o métodos de evaluación que promuevan un aprendizaje más significativo, de forma que se obtengan, consecuentemente, mejores resultados académicos.

\section{Objetivos}

En este trabajo se describe la puesta en práctica de un modelo de evaluación que denominamos “De Doble Evaluación" (DDE), el cual tiene en cuenta la dimensión formativa de la evaluación. Además, se analizan los resultados de su puesta en práctica en la asignatura Matemáticas, del Grado de Ciencias Ambientales de la Universitat Politècnica de València (GCCAA-UPV). En concreto, se quiere verificar si se producen mejoras en el rendimiento académico de los alumnos y si, con el sistema de evaluación, también se influye positivamente en la actitud de los alumnos hacia las matemáticas y, consecuentemente, en el interés de los alumnos por aprenderlas.

\section{Descripción y desarrollo de la innovación}

\subsection{Descripción}

El modelo DDE es simple: tras haber trabajado una parte de la materia, se realiza un primer control de evaluación. El resultado de dicho control informa a alumno y profesor del nivel de adquisición de competencias alcanzado. El siguiente paso es desarrollar actividades formativas orientadas a subsanar las lagunas competenciales y errores detectados en el primer control, dando de esta forma feedback al alumno. Finalmente se realiza un segundo control de evaluación, cuyo resultado proporcionará una nueva valoración del nivel competencial del alumno en esa parte concreta de la materia (figura 1).

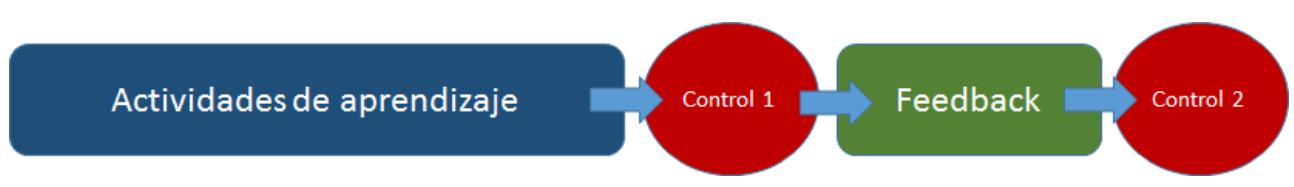

Fig.1 Esquema del modelo DDE 
El modelo DDE se fundamenta en el hecho de que es normal cometer errores durante y después del proceso de aprendizaje de algo nuevo (Huitrado y Climent, 2013; Rico, 1998; Socas, 2007). Más aún, cometer errores es una parte natural del proceso de aprendizaje y, en el caso de las matemáticas, los errores cometidos proporcionan al alumno información muy valiosa sobre el nivel competencial alcanzado, y lo que es más importante, nuevas oportunidades de aprendizaje con un conocimiento más claro de aquello que no se sabe o no se sabe hacer.

Los resultados del primer control también van a ser muy importantes para los profesores ya que informarán sobre aquello que no ha funcionado en el proceso de enseñanza/aprendizaje inicial. Los errores de los alumnos constituyen un indicador inestimable para verificar en qué aspectos el profesor debe profundizar o poner más énfasis, qué explicaciones vale la pena repetir o qué estrategias de enseñanza es conveniente revisar o cambiar. En definitiva, se trata de "aprovechar" los errores iniciales de los alumnos, identificándolos oportunamente a partir del primer control de evaluación, para convertirlos en nuevas oportunidades en favor del aprendizaje. Obviamente, sería deseable que las sesiones de feedback se desarrollen de forma que se proporcione a los alumnos solamente la mínima ayuda, aquella estrictamente necesaria para que sean ellos, por ellos mismos, los que subsanen los errores y resuelvan los problemas. Sin embargo esto último necesita de un tiempo del que, en general, no se dispone en la universidad, teniéndose que recurrir al desarrollo de actividades bastante dirigidas.

\subsection{Participantes}

En la experiencia participaron 61 estudiantes de primer curso del Grado en Ciencias Ambientales, del Campus de Gandia de la Universitat Politècnica de València, durante el primer cuatrimestre del curso 2017/2018. Todos ellos cursaban la asignatura Matemáticas $\mathrm{y}$, como viene siendo habitual, formaban un grupo muy heterogéneo si se tiene en cuenta lo estudiado en el curso anterior (diversos bachilleratos, ciclos formativos y repetidores). Por esta razón, al observar los conocimientos matemáticos previos en el grupo, nos encontramos con un número importante de alumnos que en $2^{\circ}$ de bachillerato no cursaron matemáticas, alumnos procedentes de un ciclo formativo profesional así como un número significativo de alumnos repetidores.

Si fijamos el objetivo en la manera en que los estudiantes hacen frente al estudio de las matemáticas, resultan más que evidentes ciertas actitudes poco convenientes: asistencia irregular a las clases, manifiesta incompetencia a la hora de elaborar sus propios apuntes y realización de las tareas de aprendizaje de forma irregular en el tiempo, como la preparación de los exámenes a última hora. Pero quizás lo más destacable sea una actitud previa hacia las matemáticas que, en general, cuando no es negativa es de indiferencia. En base a los elementos contextuales anteriores, es inmediato prever un índice alto de fracaso en las asignaturas de matemáticas. Por lo tanto se impone actuar para evitar en la medida de lo posible el fracaso, al tiempo que se pueda facilitar un aprendizaje significativo.

(cc) EY-NC-ND 2018, Universitat Politècnica de València

Congreso IN-RED (2018) 


\subsection{Temporalización de la innovación}

El primer cuatrimestre del curso académico tiene una duración media de 14 semanas y en cada semana la asignatura Matemáticas tiene asignadas dos clases de teoría/problemas de 90 minutos. El periodo de 14 semanas se estructuró en dos periodos de igual duración (7 semanas cada uno). Durante el primer periodo, a lo largo de cinco semanas, se impartieron una serie de contenidos (teoría y problemas). Dicho periodo finalizó con un control o acto de evaluación. A la vista de los resultados de éste, la sexta semana se inició repasando aquellos contenidos que fueron detectados como más problemáticos para los alumnos. Además, se suministraron varios modelos de exámen para que los alumnos los fuesen realizando. La última sesión del primer periodo se dedicó a la realización de otro control o acto de evaluación. En el $2^{\circ}$ periodo del curso se estableció una distribución de clases y controles análoga a la seguida primer periodo (ver tabla 1 ).

Tabla 1. Estructura temporal de los periodos de cada cuatrimestre del grupo de innovación

\begin{tabular}{|c|c|c|}
\hline & 1a clase semanal & 2a clase semanal \\
\hline 1a y 8 a Semana & Teoria/Problemas & Teoría/Problemas \\
\hline 2a y 9a Semana & Teoria/Problemas & Teoría/Problemas \\
\hline 3a y 10 a Semana & Teoria/Problemas & Teoría/Problemas \\
\hline 4aㅡ y 11a Semana & Teoria/Problemas & Teoría/Problemas \\
\hline 5a y 12 a Semana & Teoria/Problemas & Control 1 (C1) \\
\hline 6a y 13a Semana & Repaso/feedback & Repaso/feedback \\
\hline 7ạ y 14á Semana & Repaso/feedback & Control 2 (C2) \\
\hline
\end{tabular}

La revision del primer control y las sesiones de repaso proporcionan feedback al estudiante, de forma que éste puede revisar lo aprendido y no aprendido. En el segundo control el estudiante tiene la oportunidad de mejorar la valoración obtenida en el primer control, en base a un aprendizaje más efectivo de los contenidos. La nota final otorgada al alumno para cada una de las partes fue la máxima nota al considerar los dos controles (max(Nota C1, Nota C2)). 
La doble evaluación: un medio para aprender de los errores y para mejorar el rendimiento en matemáticas

\subsection{Contenidos}

Los contenidos desarrollados en el primer periodo (7 semanas) corresponden a tópicos propios del cálculo diferencial. El segundo periodo se dedicó al cálculo integral. En la tabla 2 se exponen con más detalle los contenidos desarrollados.

Tabla 2. Estructura temporal de los periodos del primercuatrimestre

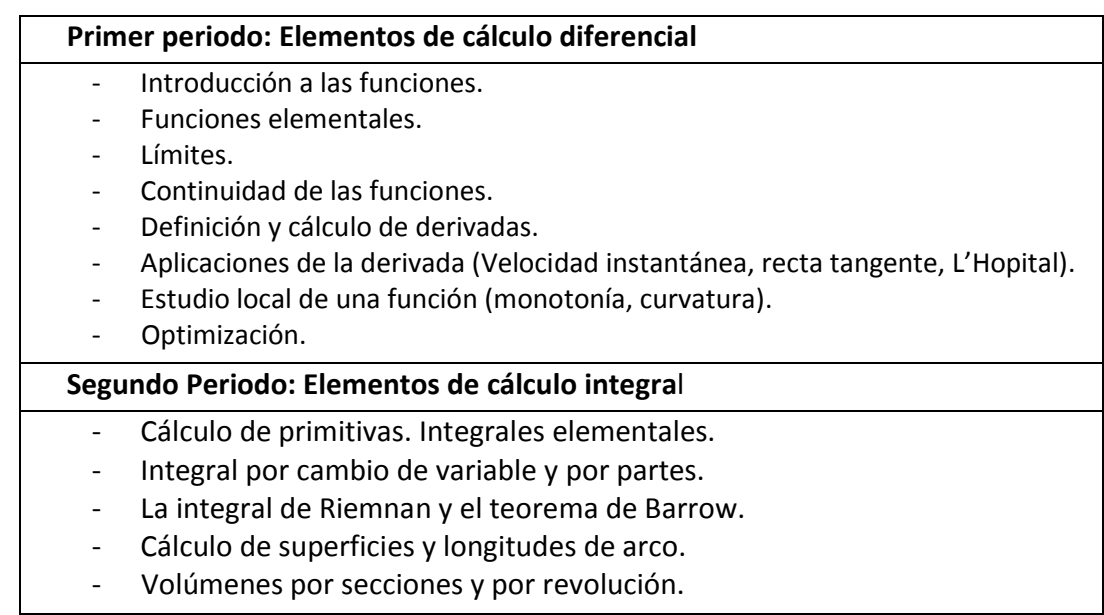

\section{Resultados y discusión}

El análisis de resultados del primer cuatrimestre del curso 2017/2018 se realiza a partir de las distribuciones de las notas correspondientes a los controles, los cuales se han valorado entre 1 y 10. Como elemento de control se tienen los resultados correspondientes al curso 2016/2017. En dicho curso, en el primer cuatrimestre, también se diferenciaron dos partes correspondientes a cálculo diferencial y cálculo integral y se siguió una metodología de evaluación, que denominaremos "tradicional”, consistente en un control para cada parte (cálculo diferencial y cálculo integral) durante el curso y la posibilidad de recuperar las partes no aprobadas (sólo una o las dos) en una única sesión de examen, al final del cuatrimestre, en la que se diferenciaban las dos partes a recuperar. Los controles consistieron, en todos los casos, en 5 preguntas de respuesta abierta con un nivel de dificultad similar.

En primer lugar se analizarán los resultados de la parte de cálculo diferencial, en concreto los correspondientes al primer control del curso 2017/2018 que se comparan con los del control de cálculo diferencial del curso 2016/2017. Seguiremos con el estudio de los resultados del segundo control de cálculo diferencial del curso 2017/2018 que se compararán con los resultados de la recuperación de cálculo diferencial, en el examen final, del curso 2016/2017. A continuación, se realiza un análisis análogo al descrito anteriormente, pero en este caso se estudian los resultados de la parte dedicada al cálculo integral. Finalmente, se compara la tasa de aprobados en todos los controles objeto de estudio.

(cc) EY-NC-ND 2018, Universitat Politècnica de València

Congreso IN-RED (2018) 


\subsection{Primera parte: Cálculo diferencial}

En la tabla 3 y la figura 2 se resume la distribución de los resultados (frecuencias absolutas, f.a. y frecuencias relativas, f.r.), por grupos de notas, y los valores promedio y de dispersión (desviación típica y coeficiente de variación, C.V.) del primer control del curso 2017/2018 (C1/CD-17) y del primer control parcial del curso 2016/2017 (C1/CD-16) que corresponden a cálculo diferencial. Los resultados evidencian las dificultades de los estudiantes de los dos cursos frente a su primer examen de matemáticas en la universidad. En ambos cursos sólo aprueban alrededor del 13\%, y además en un porcentaje bastante elevado la nota está por debajo del 2’5. Se observa también que, en ambos cursos, la nota media es muy baja, y la dispersión bastante alta, si se la compara con la media, sobre todo en el curso 2016/2017 (ver valores de C.V. en la tabla 3).

Tabla 3. Cálculo diferencial. Distribución de los resultados del primer control para los cursos 2016/2017 (C1/CD-16) y 2017/2018 (C1/CD-17)

\begin{tabular}{|c|c|c|c|c|}
\hline Intervalos & f.a. C1/CD-16 & f.a. C1/CD-17 & f.r. C1/CD-16 & f.r. C1/CD-17 \\
\hline$\left[0,2^{\prime} 5[\right.$ & 39 & 29 & $72,2 \%$ & $50,0 \%$ \\
\hline$\left[2^{\prime} 5,5[\right.$ & 8 & 22 & $14,8 \%$ & $37,9 \%$ \\
\hline$\left[5,7^{\prime} 5[\right.$ & 6 & 7 & $11,1 \%$ & $12,1 \%$ \\
\hline$\left[7^{\prime} 5,10\right]$ & 1 & 1 & $1,9 \%$ & $1,7 \%$ \\
\cline { 2 - 4 } Total & 54 & 59 & \multicolumn{3}{l}{} \\
\cline { 2 - 5 } & \multicolumn{5}{r|}{}
\end{tabular}

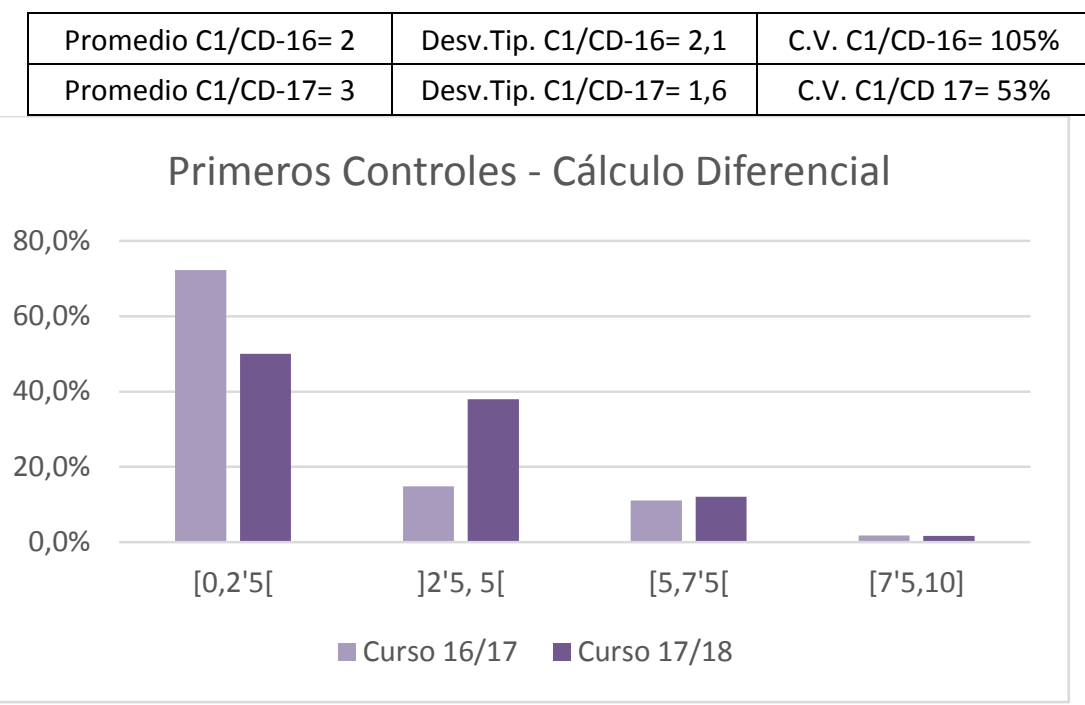

Fig. 2 Diagrama de barras de la distribución de notas del primer control del cálculo diferencial para los dos cursos

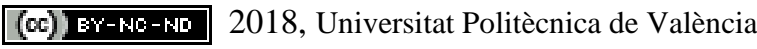




\subsection{Calculo diferencial. Control 2 (2017/2018) y examen final (2016/2017)}

Las distribuciones de los resultados para cálculo diferencial del control 2 del curso 2017/2018 (C2/CD-17) y del examen final de cálculo diferencial del curso 2016/2017 (EF/CD-16) (tabla 4 y gráfico 3) indican que los dos grupos obtienen un porcentaje de aprobados similar (30\% aprox.). Siguen siendo resultados pobres pero en el grupo DDE la nota media es más alta, el porcentaje de estudiantes que estan en el intervalo más bajo de nota es bastente menor y la dispersión respecto a la media también es mucho menor (ver valores de C.V. en la tabla 4).

Tabla 4. Cálculo diferencial. Distribución de los resultados del segundo control del curso 2017/2018 (C2/CD-17) y del examen final del curso 2016/2017 (EF/CD)

\begin{tabular}{|c|c|c|c|c|}
\hline Intervalos & f.a. EF/CD-16 & f.a. C2/CD-17 & f.r. EF/C-16 & f. r. C2/CD-17 \\
\hline$\left[0,2^{\prime} 5[\right.$ & 22 & 8 & $40,7 \%$ & $13,3 \%$ \\
\hline$\left[2^{\prime} 5,5[\right.$ & 15 & 33 & $27,8 \%$ & $55,0 \%$ \\
\hline$\left[5,7^{\prime} 5[\right.$ & 16 & 16 & $29,6 \%$ & $26,7 \%$ \\
\hline$\left[7^{\prime} 5,10\right]$ & 1 & 2 & $1,9 \%$ & $3,3 \%$ \\
\cline { 2 - 4 } Total & 54 & 59 & \multicolumn{3}{r}{} \\
\cline { 2 - 5 } & \multicolumn{4}{|r}{}
\end{tabular}

\begin{tabular}{|l|l|l|}
\hline Promedio EF/CD-16=3,4 & Desv. Tip. EF/CD-16=2,5 & C.V. EF/CD-16=73.5\% \\
\hline Promedio C2/CD-17=4,3 & Desv. Tip. C2/CD-17=1,6 & C.V. C2/CD-17=37.2\% \\
\hline
\end{tabular}

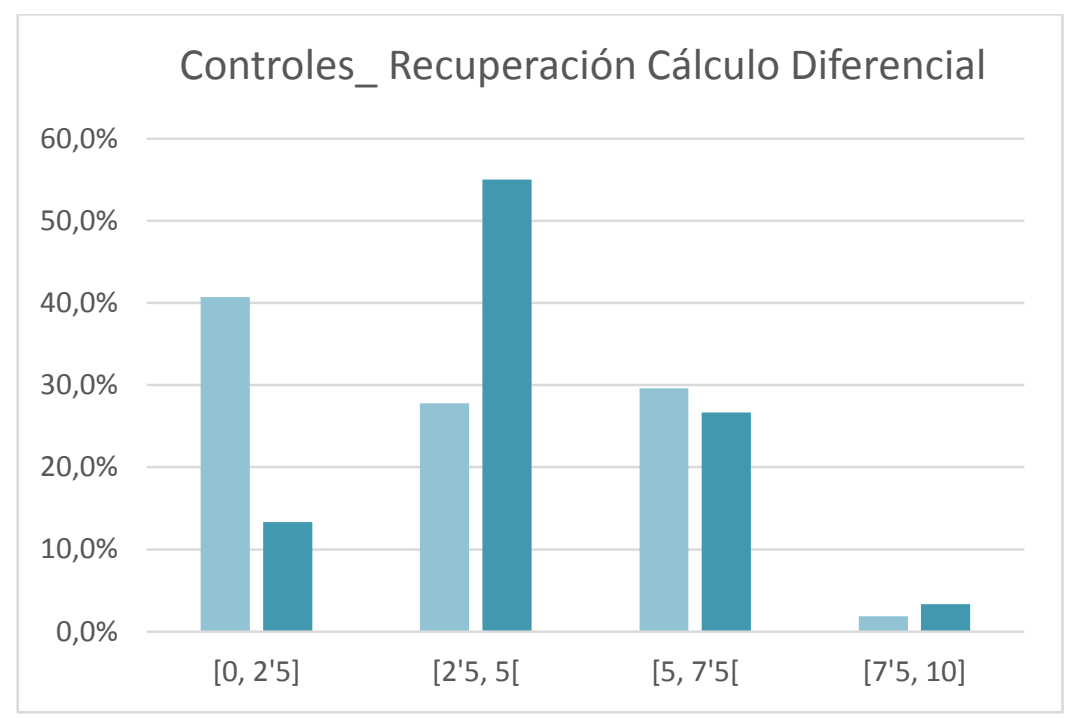

Fig. 3 Diagrama de barras de la distribución de las notas en $C 2 / C D$ (Curso 17/18) y EF/CD (Curso 16/17) 


\subsection{Segunda parte: Cálculo integral}

En la tabla 5 y el gráfico 4 se resumen los resultados, por grupos de notas, del primer control de cálculo integral del curso 2017/2018 (C1/CI-17) y del control parcial de la misma parte del curso 2016/2017 (C1/CI-16). El grupo que sigue una evaluación tradicional (curso 2016/2017), tiene unos resultados similares a los obtenidos en C1/CD-16. En cambio, en el grupo con evaluación DDE se incrementa la tasa de aprobados, estableciéndose una diferencia notable en el rendimiento al comparar los dos grupos. La diferencia entre la nota media de los dos grupos es de más de 2 puntos.

Tabla 5. Cálculo integral. Distribución de los resultados del primer control para los cursos 2016/2017 (C1/CI-16) y 2017/2018 (C1/CI-17)

\begin{tabular}{|c|c|c|c|c|}
\hline Intervalos & f.a. C1/Cl-16 & f.a. C1/Cl-17 & f.r. C1/Cl-16 & f.r.C1/Cl-17 \\
\hline$\left[0,2^{\prime} 5[\right.$ & 22 & 8 & $57,9 \%$ & $16,7 \%$ \\
\hline$\left[2^{\prime} 5,5[\right.$ & 9 & 17 & $23,7 \%$ & $35,4 \%$ \\
\hline$\left[5,7^{\prime} 5[\right.$ & 5 & 16 & $13,2 \%$ & $33,3 \%$ \\
\hline$\left[7^{\prime} 5,10\right]$ & 2 & 7 & $5,3 \%$ & $14,6 \%$ \\
\cline { 2 - 4 } Total & 38 & 48 & \multicolumn{1}{l}{} \\
\cline { 1 - 4 } & \multicolumn{4}{|r}{}
\end{tabular}

\begin{tabular}{|l|l|l|}
\hline Promedio $\mathrm{C} 1 / \mathrm{Cl}-16=2,6$ & Desv. Tip. $\mathrm{C} 1 / \mathrm{Cl}-16=2,5$ & C.V. $\mathrm{C} 1 / \mathrm{Cl}-16=96.15 \%$ \\
\hline Promedio $\mathrm{C} 1 / \mathrm{Cl}-17=4,9$ & Desv. Tip. $\mathrm{C} 1 / \mathrm{Cl}-17=2,4$ & C.V. $\mathrm{C} 1 / \mathrm{Cl}-17=48.98 \%$ \\
\hline
\end{tabular}

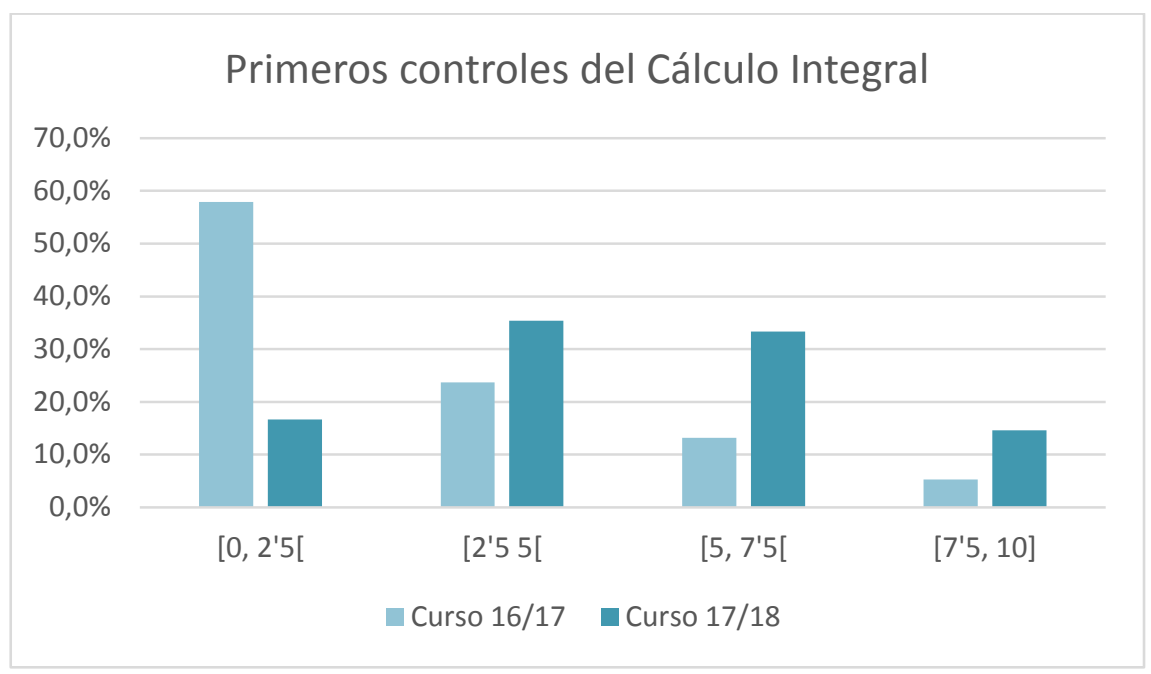

Fig. 4 Diagrama de barras de la distribución de notas del primer control de cálculo integral para los dos cursos 
4.4 Cálculo Integral. Control 2 (2017/2018) y examen final (2016/2017)

Según los resultados que se resumen en la tabla 6 y el gráfico 5, el grupo DDE presenta una tasa de aprobados más alta que el grupo con evaluación tradicional. La diferencia en el promedio de las notas supera los dos puntos. Además, sigue habiendo en el grupo DDE un transvase de estudiantes desde los intervalos de notas más bajas hacia intervalos de notas más altas.

Tabla 6. Cálculo integral. Resultados del segundo control del curso 2017/2018 (C2/CI-17) y del examen final del curso 2016/2017 (EF/CI-16)

\begin{tabular}{|c|c|c|c|c|}
\hline Intervalos & f.a. EF/Cl-16 & f.a. C2/Cl-17 & f.r. EF/Cl-16 & f.r. C2/Cl-17 \\
\hline$\left[0,2^{\prime} 5[\right.$ & 20 & 5 & $50,0 \%$ & $9,8 \%$ \\
\hline$\left[2^{\prime} 5,5[\right.$ & 9 & 19 & $22,5 \%$ & $37,3 \%$ \\
\hline$\left[5,7^{\prime} 5[\right.$ & 9 & 20 & $22,5 \%$ & $39,2 \%$ \\
\hline$[7,5,10]$ & 2 & 7 & $5,0 \%$ & $13,7 \%$ \\
\hline \multirow{2}{*}{ Total } & 40 & 51 & & \\
\cline { 2 - 5 } & & &
\end{tabular}

\begin{tabular}{|l|l|c|}
\hline Promedio $\mathrm{EF} / \mathrm{Cl}-16=3,00$ & Desv. Tip. $\mathrm{EF} / \mathrm{Cl}-16=2,5$ & C.V. $\mathrm{EF} / \mathrm{Cl}-16=83.33$ \\
\hline Promedio $\mathrm{C} 2 / \mathrm{Cl}-17=5,13$ & Desv. Tip. $\mathrm{C} 2 / \mathrm{Cl}-17=2,2$ & C.V. $\mathrm{C} 2 / \mathrm{Cl}-17=42.88 \%$ \\
\hline
\end{tabular}

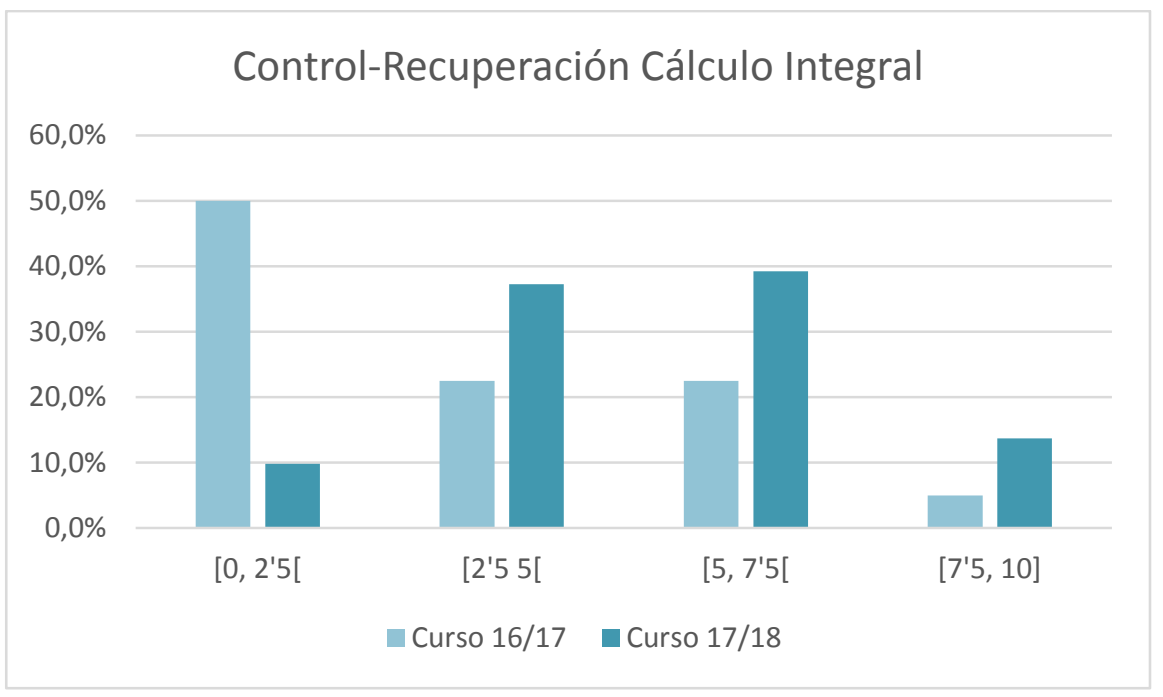

Fig. 5 Diagrama de barras correspondiente a la distribución de las notas en C2/CI (Curso 17/18) y EF/CI (Curso 16/17)

\subsection{Evolución de la tasa de aprobados}

Al observar la tasa de aprobados (aprobados/presentados) representada en la figura 6, en el curso 2016/2017 se observa una tendencia característica, que se repite curso tras curso, en la cual el cálculo integral presenta serias dificultades para los alumnos, lo cual se evidencia

(cc) EY-NC-ND 2018, Universitat Politècnica de València 
en la línea, con pendiente negativa, que corresponde al paso desde C2-EF/CD a C1/CI. En el caso de la poligonal correspondiente al DDE (curso 2017/2018) se mantiene una tendencia claramente creciente en todo el cuatrimestre, lo cual constituye una evidencia de la mejora del rendimiento, así como de la actitud del alumnado frente a la asignatura. Las diferencias con el grupo que seguía un sistema de evaluación tradicional son obvias.

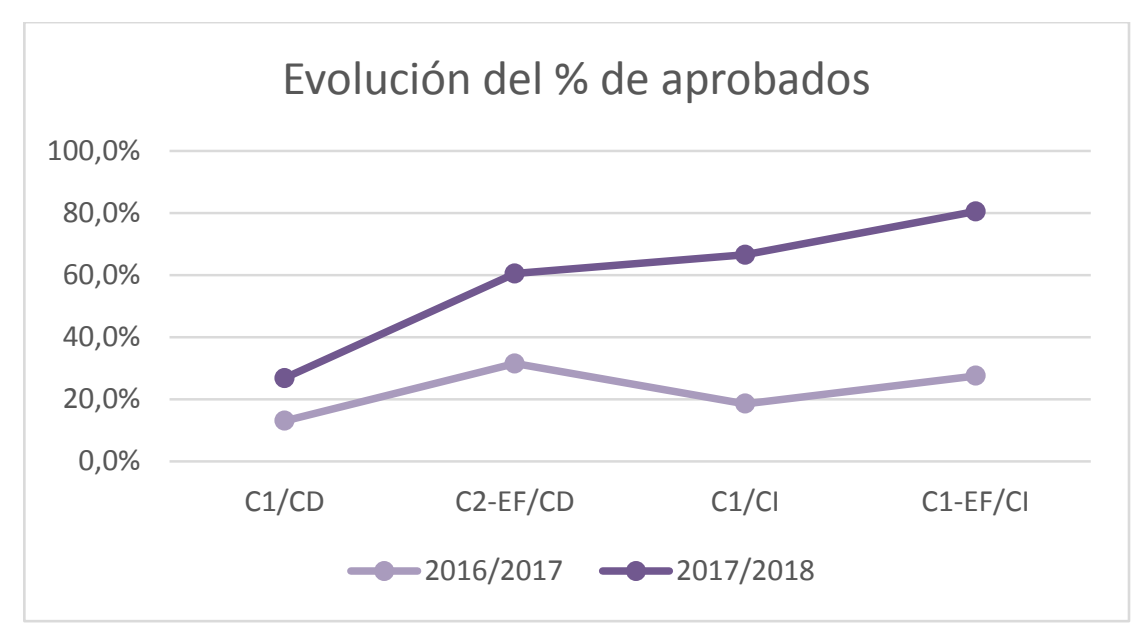

Fig. 6 Evolución de la tasa de aprobados a lo largo del cuatrimestre (cursos 2016/2017 y 2017/2018).

\section{Conclusiones}

Los resultados del análisis realizado ponen en evidencia las dificultades que los estudiantes tienen con las matemáticas al acceder a estudios universitarios. En el primer examen (C1/CD-16 y C1/CD-17), se observa claramente que hay un elevado número de estudiantes que no solo no aprueban, sino que obtienen notas muy bajas, tanto en el grupo con evaluación tradicional como el DDE. El paso de la educación secundaria a la universitaria sigue siendo complicado en matemáticas, especialmente para estudiantes de grados científicos.

Con el sistema de doble evaluación, o DDE, (Enseñanza+Evaluación 1+Refuerzo (feedback)+Evaluación 2) se consigue cierta mejora en el rendimiento, pasando de un $12 \%$ a un 53\% de aprobados, y además, el porcentaje de los que estaban en el nivel más bajo de notas pasa de un $50 \%$ a solo un $9{ }^{\prime} 5 \%$. Si se comparan estos resultados con los obtenidos en el grupo con sistema de evaluación trdicional, el progreso resulta evidente.

Teniendo en cuenta el contexto, las características de los grupos y los resultados, se considera que dicha mejora se debe principalmente al cambio en la metodología de evaluación empleada, que se caracteriza sobre todo por reforzar el aprendizaje de los contenidos a partir de los errores y deficiencias observadas en el primer control, y no esperar demasiado tiempo para que los alumnos puedan demostrarse a si mismos que las matemáticas son accesibles y, por lo tanto, que es posible mejorar los resultados. 
La doble evaluación: un medio para aprender de los errores y para mejorar el rendimiento en matemáticas

\section{Referencias}

FABREGAT, J. y PELAYO, I. (2016).”Plan inicial, progresos explícitos y evaluación de una trama de feedforwarding sobre campus virtual en una asignatura de matemáticas” en IN-RED 2016 Congreso de Innovación Educativa y Docencia en Red de la Universitat Politècnica de Valencia. Valencia. Disponible en http://ocs.editorial.upv.es/index.php/INRED/INRED2016/paper/view/4404 [Consulta: 1 de febrero de 2018].

GARGALLO, B. GARCÍA E., MORERA I. y BENAVENT A. (2015). "Métodos innovadores y enfoques de aprendizaje en estudiantes” en IN-RED 2015 Congreso de Innovación Educativa y Docencia en Red de la Universitat Politècnica de Valencia. Valencia. Disponible en http://dx.doi.org/10.4995/INRED2015.2015.1576 [Consulta: 30 de enero de 2018].

HABERYAN, K. A. (2003) "Do weekly quizzes improve student performance on general biology exams?” The American Biology Teachers, 65, p. 110-114..

HERNÁNDEZ SÁNCHEZ, A.A., (2010). "Necesidad de la evaluación en el proceso de aprendizaje” en monografías.com Disponible en http://www.monografias.com/trabajos80/ necesidad-evaluacion-proceso-aprendizaje/necesidad-evaluacion-proceso-aprendizaje.shtml [Consulta: 1 de febrero de 2018].

HUITRADO, J. L. y CLIMENT, N. (2013). Conocimiento profesional del profesor ante errores relativos al álgebra de los alumnos de secundaria. En A. Berciano, G. Gutiérrez, A. Estepa y N. Climent (Eds.), Investigación en Educación Matemática XVII (pp. 327-336). Bilbao: SEIEM.

ISABEL, A. y VARGAS, M. (2004). La Evaluación Educativa: Concepto, Períodos y Modelos. Actualidades Investigativas en Educación, 4(2), p. 1-28. http://doi.org/10.15517/aie.v4i2.9084

MOURSHED, M., CHIJIOKE, C. y BARBER M. (2010). How the world's most improved school systems keep getting better. London: McKinsey y Company. Disponible en http://hub.mspnet.org/index.cfm/22017 . [Consulta: 30 de enero de 2018]

MYERS, C. B. y MYERS, S. M. (2007). “Assenssing assessment: The effects of two exam formats on course achievement and evaluation” en Innovative Higher Education, 31, p. 227-236.

RICO, L. (1998). Errores y dificultades en el aprendizaje de las matemáticas. En J. Kilpatrick et al (Eds.), Educación Matemática: Errores y dificultades de los estudiantes, Resolución de problemas, Evaluación e Historia (pp. 69-108). Bogotá: Una Empresa Docente.

SADLER, P. M. y GOOD, E. (2006). "The impact of self-and peer-grading on student learning”. Educational assessment, vol. 11, 1, p. 1-31.

SIMON, M. A. y TZUR, R. (2004). Explicating the Role of mathematical Tasks in conceptual Learning: An Elaboration of the Hypothetical Learning Trajectory. Mathematical Thinking and Learning, 6(2), p.91-104.

SOCAS, M. (2007). Dificultades y Errores en el Aprendizaje de las Matemáticas. Análisis desde el Enfoque Lógico Semiótico. En M. Camacho, P. Flores y M.P. Bolea (Eds.), Investigación en Educación Matemática XI (pp. 19-52). Tenerife: SEIEM.

TYLER, R.W. (1974). Principios básicos del currículo. Buenos Aires, Troquel

ZABALZA, M.A. (2004).” Innovación en la enseñanza universitaria” en Contextos Educativos, 6-7, p. 113- 136

(cc) EY-NC-ND 2018, Universitat Politècnica de València

Congreso IN-RED (2018) 